\title{
DEVELOPMENT OF A HARDWARE AND SOFTWARE SYSTEM FOR METEOROLOGICAL OBSERVATIONS
}

\author{
Mikhail Shcherbakov*, Alexey Borisov \\ Altai State Technical University, 656000, Barnaul, Russia
}

\begin{abstract}
Article is devoted to development of a hardware and software system for monitoring of weather conditions. The hardware of this complex is the Arduino platform, which are connected sensors of temperature, humidity, atmospheric pressure, speed and direction of wind, level of ultra-violet radiation, which allow to watch environment parameters. Data are transferred to the remote server which is constructed on the basis of the single board computer Raspberry Pi, by means of a radio channel. For ensuring functioning of a complex, the software was written and it consisting of three programs. The first program was written to on Si similar language for operation of the Arduino controller. The second from programs was written for functioning of the server, it consists of MySQL DBMS, a script for data record in a basis and the PhpMyAdmin servers for administration of a DB. The third was written in the C\# programming language and represents the application Windows forms. The made experiment showed that the complex allows to carry continuously meteorological observations, at the same time data remain in the database and the consumer software allows to carry their processing.
\end{abstract}

\section{Introduction}

The existing meteorological stations have changing for more than 100 years. At the beginning of the 20th century they represented laboratories, with devices for meteorological observations which were controlled by the person. Now it is almost completely the automated complexes which are collecting data on the environment practically without intervention of a person and giving results of observations already in the processed look.

Automatic meteorological stations with, partial cycle of observations (only collection of information, storage and/or it transfer) are widespread. They are used for expansion of a zone of observation of metrological stations or control of weather conditions in certain territories, for example, the airports, production sites, etc. [1].

The theory and practice of application of similar hardware and software systems showed their effectiveness. They usually are autonomous, simple in using, suitable for carrying out the researches which aren't demanding excessive accuracy, but conducted a long time.

\footnotetext{
* Corresponding author: shermih1996@mail.ru
} 


\section{Relevance}

Nowadays almost each person learns temperature or watching a weather forecast per day before an exit from the house that put on according to weather conditions. That is why the problem of obtaining of current information on state of environment haven't an equal relevance.

Presently the state and commercial systems of monitoring weather conditions and their forecasting are developed, but their accuracy in various territories differ and in some places the error of measurements and forecasts is high. It is connected with the fact that now the shortage of posts of meteorological observations is observed in Western Siberia and in some other regions of Russia that leads to a lack of information for creation of the exact forecast of weather conditions. For example, in Altai Krai there are only about 30 posts for meteorological observations which are widely spaced and located unevenly [2]. In this regard in Russia automatic meteorological stations receive the increasing popularity. They are used by the commercial organizations, groups of researchers or ordinary people [3].

They differ in usability, mobility, a set of complete sets. Their functionality depends on the price. Meteorological stations with broad functionality differ in high cost. It does their acquisition difficult by enterprises with has small capital, some higher educational institutions, or research groups. This fact does development of an automatic meteorological station with the low cost and broad functionality very relevant.

\section{Work algorithm}

The automatic meteorological station is used for meteorological observations without participation of people. It provides the systematized data on the made observations and it usually is partially or completely autonomous.

The meteorological station consists of two principal elements: data acquisition unit and server.

The data acquisition unit consists of a set of sensors, for collection of meteodata, the controller of control, the interface of connection and power supply unit. All elements are in the tight cases. Treat sensors:

1. Barometer. The meteorological station measures atmospheric pressure by means of this device.

2. Thermometer. It takes air temperature.

3. Hygrometer. The device defines humidity of air.

4. Weather vane. The device shows the direction of wind.

5. Anemometer. The device for fixing of force and speed of streams of wind.

6. Sensor of level of ultra-violet radiation. It measures quantity of the solar radiation reaching the Earth's surface.

7. Sensor of an amount of precipitation. It measures the level of precipitations.

The controller controls of sensors. It realizes data collection, their transfer on the server and power management controls sensors.

All parameters from sensors are transferred to the server. The server stores measurements in the database, reports results in the form of tables, diagrams, etc. General algorithm of work of a hardware and software system:

1. The block of data collection located on the street by means of sensors, conducts monitoring of conditions of the environment;

2. The controller polls all sensors and collects from them information one time during a certain time frame:

3. The controller transfers data to the server on wireless connection by means of a radio channel: 
4. The server writes data in the database.

5. A user connects the personal computer to the server by means of the Wi-Fi network. By means of the special software it is connected to the database of the server, and it performs the data operations provided by a software functionality.

The scheme of action of system is submitted in the Figure 1.

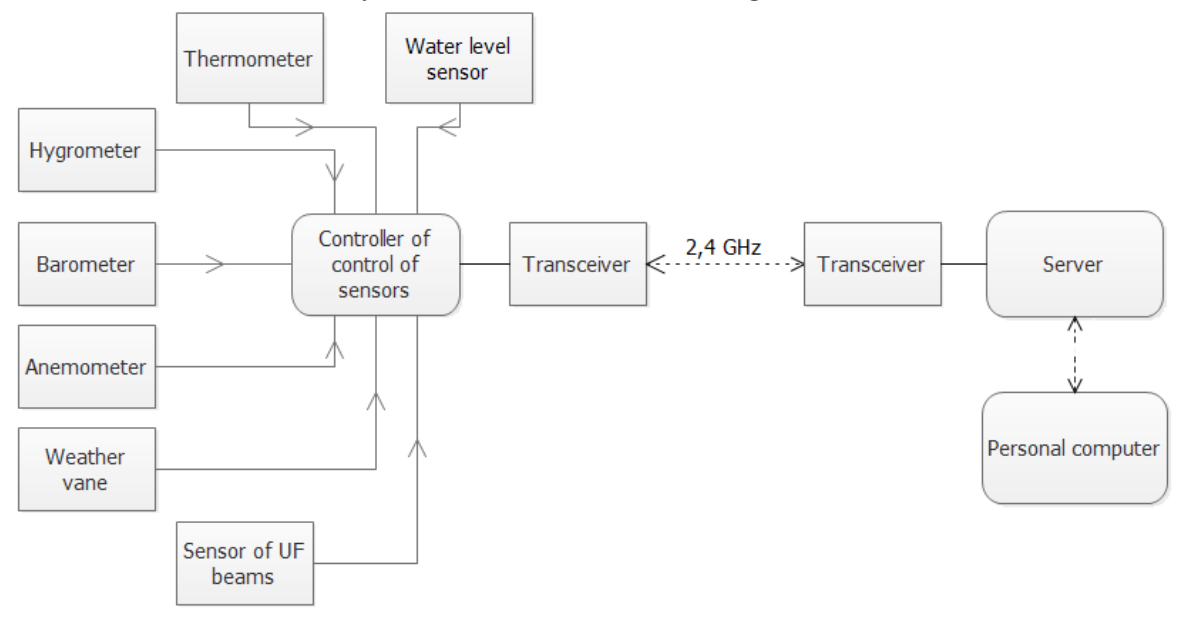

Fig. 1. Scheme of action of system.

\section{Hardware-software providing}

As it was already provided in the previous point, for monitoring the following parameters of the environment are selected: temperature, air humidity, atmospheric pressure, level of an ultraviolet light, direction and wind speed, level of precipitates.

The acquisition unit keeps their monitoring of these parameters. The appropriate sensors are used for monitoring of these parameters. By means of real-time clocks exact time of data collection is marked. A supply of the unit is carried out from an external source and/or the built-in power supply (the accumulator and a solar panel). All modules are run by the controller [4].

For the block of data collection the following components are used:

- microcontroller Arduino Mega 2560. Clock rate of the processor $16 \mathrm{MHz}$, the RAM of $8 \mathrm{~KB}$, ROM of $4 \mathrm{~KB}$, a working tension 7-12B, the consumed current up to $90 \mathrm{~mA}$;

- sensor of temperature and humidity DHT22, range of temperatures is $-40-125( \pm 0.2)$

${ }^{\circ} \mathrm{C}$, humidity of air $0 \%-100$ of $\%( \pm 2 \%)$, max. current $2.5 \mathrm{~mA}$, measurement up to $0.5 \mathrm{~Hz}$ (times in 2 seconds), working tension $3-5 \mathrm{~V}$;

- atmospheric pressure sensor (barometer) BME280, operating range: 300-1100 GPa, an error of $0.2 \mathrm{~Pa}$, an error of sensitivity of $\pm 0,25 \%$, the maximum consumed current $3.5 \mathrm{mkA}$;

- the sensor of ultra-violet (UF) radiation GUVA-S12SD, the range of length of the found waves of 240-370 nanometers, viewing angle of 130 degrees, the consumed current of $1 \mathrm{~mA}$;

- sensor of quality of air MQ-135, type: electrochemical, supply voltage: $5 \mathrm{~V}$, the consumed current: $150 \mathrm{~mA}$, measurement range: 0.001 - 1\% (10-1000 ppm);

- water level sensor. Working current $20 \mathrm{~mA}$, tension 3-5V;

- real-time clocks DS3231, values of the report: second, minute, hour, a day of the week, day of month, month, year, high-speed performance of the interface up to $400 \mathrm{kHz}$, are supplied with the independent power supply; 
- microSD memory card module, the memory card of the MicroSD card format, memory size is $4 \mathrm{~GB}$;

- power control unit, tension $9 \mathrm{~V}$, maximum current $1 \mathrm{~A}$;

- $\quad$ solar panel, the given tension $7.5 \mathrm{~B}$, the max. given power of $5.5 \mathrm{~W}$;

- accumulator, 5000 Ach;

- module of the wireless connection NRF24L01, transmit frequency is $2.4 \mathrm{GHz}$, range is up to 1100 meters, in urban development up to $520 \mathrm{~m}$, transmission rate up to $2 \mathrm{Mbit} / \mathrm{s}, 126$ channels for data transfer, a maximum current (in a transmission mode) $13.5 \mathrm{~mA}$, in a sleep mode $25 \mathrm{mkA}$, a working tension from 1.9 to $3.6 \mathrm{~V}$.

The server includes the following components:

- single board computer Raspberry Pi 3 B+, processor ARMv8 4x1.2 GHz, RAM 1GB, Wi-Fi, Blutooth 4.1, Ethernet port, 4 USB ports;

- module of the wireless connection NRF24L01 (the description is given above).

The flowchart of the hardware of the complex is provided in the Figure 2.

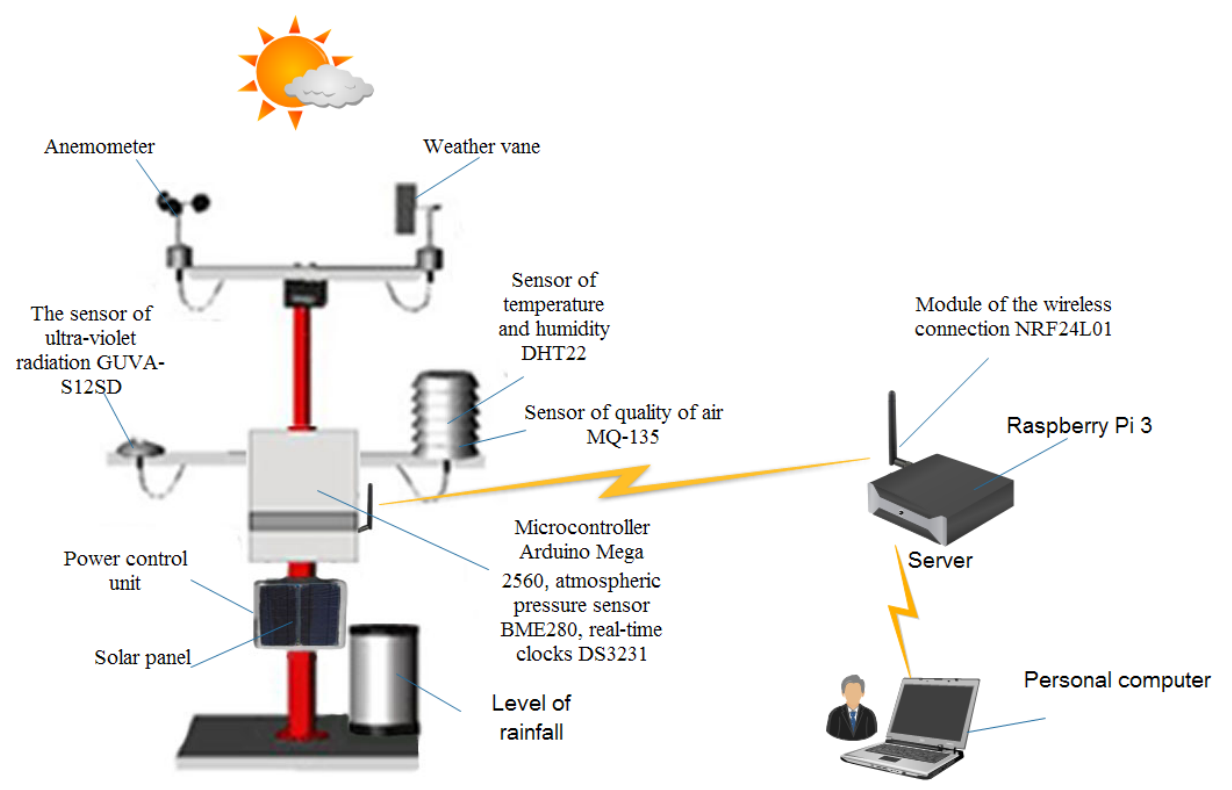

Fig. 2. Flowchart of the hardware of the complex.

The general algorithm of functioning of the hardware complex consists in the following:

1. Arduino collects environment state-of-health data from the sensors which is built in data acquisition unit;

2. Arduino adds to data the exact time taken from real-time clocks, arranges it in the form of a line and sends it on the radio channel to the server.

3. Raspberry Pi writes data in the MySQL database.

4. If it is necessary, data are sented on the third-party device on the Internet network, by means of Wi-Fi or via the RJ-45 interface.

If a user needs to get data access, it will take the following actions:

1. He will connect the personal computer to the server by means of Wi-Fi or a cable;

2. He will get access to the database, having used the special software;

3. User will perform necessary operations which are provided by the software functionality. 
The software consists of three programs: the first is written for functioning of the Arduino microcontroller, the second for creation of the server on the Raspberry Pi computer, the third for working on the personal computer.

The program for the controller is written to $C$ the similar programming language which is specially adapted for Arduino. It will execute the following functions:

- Data collection from sensors.

- Transfer of received data to a line and sending to the server.

- Data collection from management system a supply (a charge of the accumulator, a given current and tension).

- Transfer of all received data to the server.

The program for Raspberry Pi represents MySQL DBMS, the script for data record in a basis and the PhpMyAdmin servers for administration of a DB. The software performs the following operations:

- Data acquisition from data acquisition unit and their recording in the database.

- Saving data in the file in necessary format.

- Provisions of access to the database for the client.

The program for the computer is written in the form of a window Windows application in the $\mathrm{CH}$ programming language. Performs the following functions:

- Receiving of access to the database of the server.

- System status request (accumulator charge, operation of sensors).

- Execution of manipulations with information from the database according with the software functionality (an output of the current indications of sensors, statistics for a certain period, etc.).

The interface of the software is provided in a Figure 3.

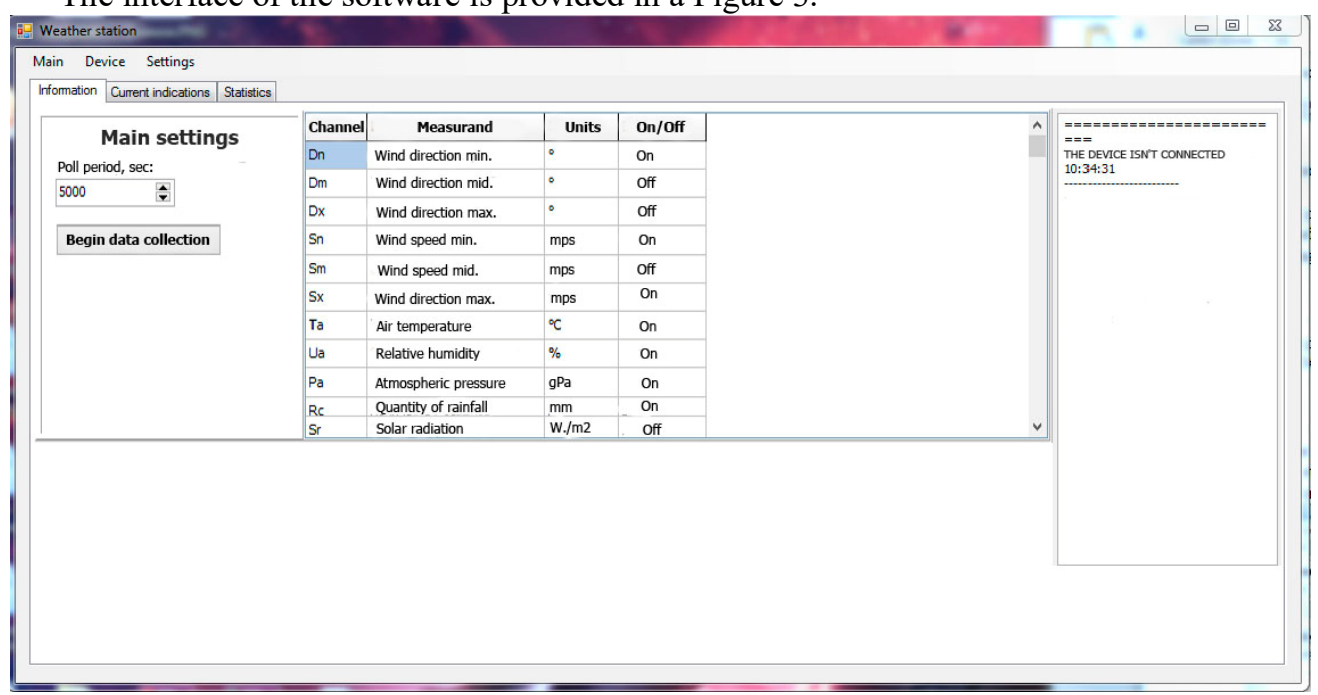

Fig. 3. Software interface.

The interface contains the following elements:

The main menu contains three points: "Principal", "Device" and "Settings".

In the "Setup" point the user has an opportunity to make system operation adjustment: the list of sensors from which data, an inquiry interval, etc.

In the "Device" point the interface removes state-of-health data of the hardware complex such as: connection of the server, data acquisition unit, status of sensors, accumulator charge, etc. 
In the "Main" point there are three subparagraphs. In the subparagraph "Information" the user has an opportunity to get acquainted with a current status of system, that is whether the PC is connected to the server what functions of system are switched on or switched off, etc. The view of a window is provided in a Figure 3.

In the subparagraph "Current indications" the dialog of a request of indications from all sensors of the unit of observation is located. The user has an opportunity to examine dataful monitoring, taken directly from the unit of observation. The single request, or information up-dating is possible each several seconds (the period of a request is specified in textbox). In a Figure 4 the interface of a dialog of a request and result of a request is provided.

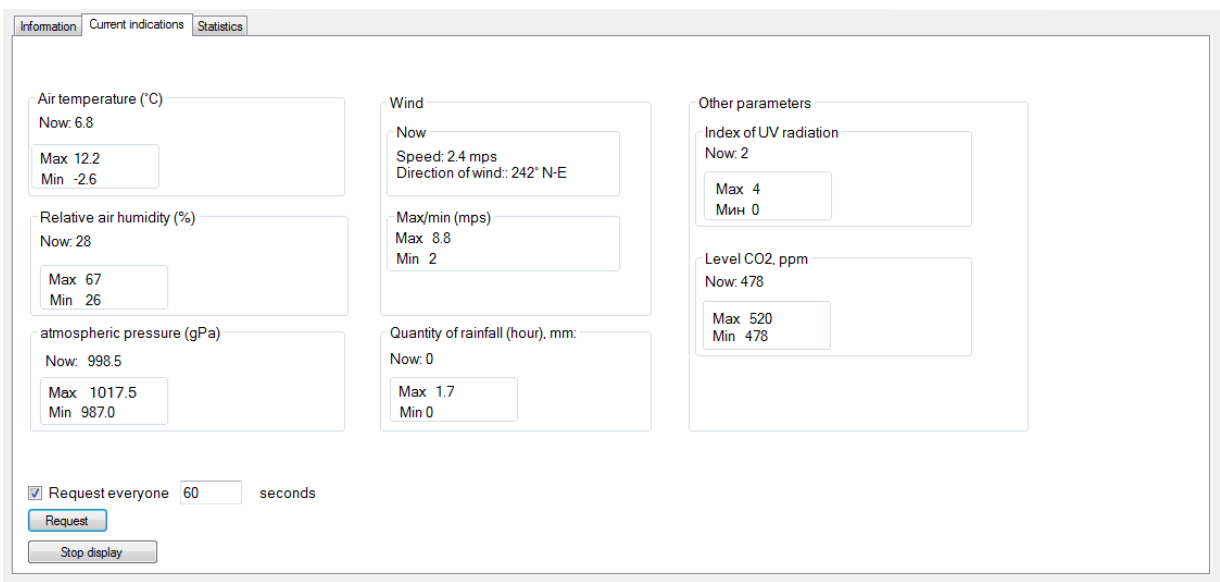

Fig. 4. Conclusion of the current indications of sensors.

The information output dialog from the database of the server in the form of the table or the diagram is posted in the section "Statistics". In the table information from all sensors for the period of measurements set by the user is output. In the form of the diagram the statistics of indications of temperature, relative air humidity, an atmospheric pressure, wind speed, the index of solar activity, impurity of $\mathrm{CO} 2$ air, for the set time frame can be requested ( 24 hours, 48 hours, 72 hours and so on). In a Figure 5 the diagram of air temperature, from the data obtained during meteorological observations an interval of record of indications of a basis of 60 minutes, the period of observations of 24 hours is provided.

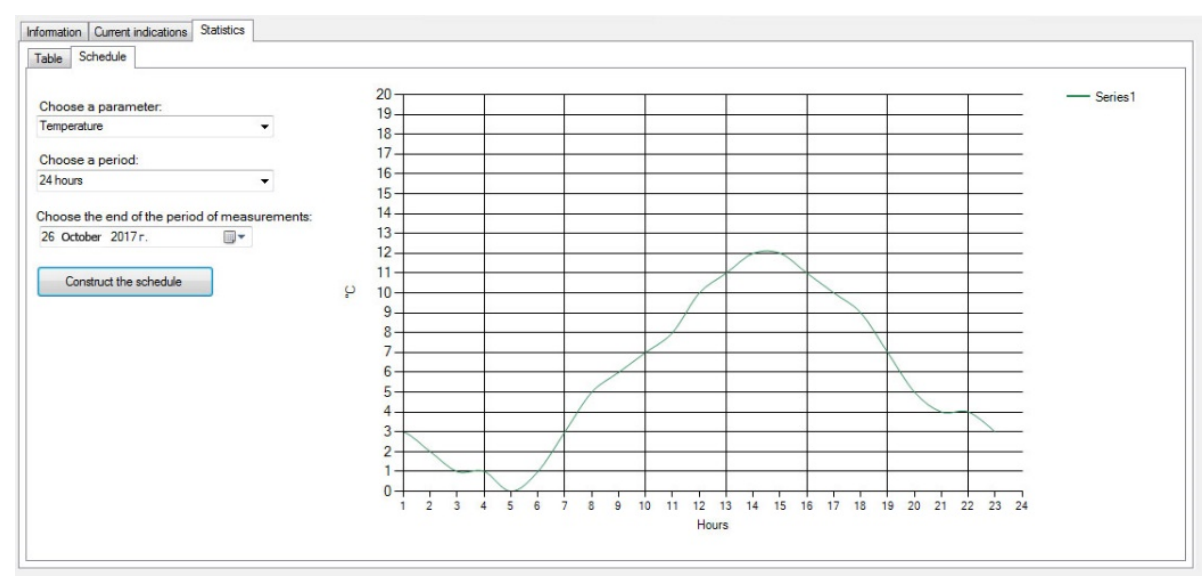

Fig. 5. The conclusion of the schedule of temperature for the set period. 
As it was told earlier, the data acquisition unit and the server communicate with each other by means of a radio channel. The radio wave in the course of distribution in space occupies volume in the form of an ellipsoid of revolution with the maximum radius in the middle of flight which is called a Fresnel zone. Natural (the earth, hills, trees) and artificial (buildings, poles) barriers getting to this space weaken a signal. For optimum operation of a meteorological station and a steady signal between modules calculation of a Fresnel zone for a radio channel was made. Radius of the first Fresnel zone is calculated with the help of the following formula

$$
R=17,3 \sqrt{\frac{1}{f} \cdot \frac{S D}{S+D}}
$$

where $R$ - the radius of a Fresnel zone (m); $S, D$ - distance from antennas to the highest point of an estimated hindrance $(\mathrm{km}) ; f$ - frequency $(\mathrm{GHz})[5]$.

During researches the distance between antennas was equal about $200 \mathrm{~m}$, distance to a hindrance of 20 meters. Result of computation:

$$
R=17.3 \sqrt{\frac{1}{2.437} \cdot \frac{20 \cdot 180}{20+180}}=26.8(\mathrm{~m})
$$

Therefore, that attenuation of a signal was minimum, devices have been placed so that the distance to an obstacle didn't come into Fresnel's zone with a radius of $26.8 \mathrm{~m}$.

\section{Conclusion}

The carried-out laboratory and field tests with use of a hardware and software system for meteorological observations showed satisfactory results. The complex allows to control over the selected environment parameters in real time, and also to carry out data analysis, collected for a certain time frame.

\section{References}

[1] M. Shcherbakov, A. Borisov, Collection of scientific articles of the III International scientific-practical 1, 185 (2017)

[2] Official site of Hydrometeorological center of Russia URL: http://www.meteoinfo.ru

[3] C. K. Wright, K. M. de Beurs, Z. K. Akhmadieva, P. Y. Groisman and G M Henebry Environ. Res. Lett. 4, 045020 (2009) doi:10.1088/1748-9326/4/4/045020

[4] M. Shcherbakov, A. Borisov, Materials of the regional youth scientific and practical conference "Program Technical Supply of Automated Systems" 1, 111 (2017)

[5] The relation "signal noise" in digital communication systems. Creation of antennafeeder paths and radio systems with external antennas URL: http://www.intuit.ru/studies/courses/1004/202/lecture/2796 